\title{
Two heterozygous mutations identified in one Chinese patient with bilateral macular coloboma
}

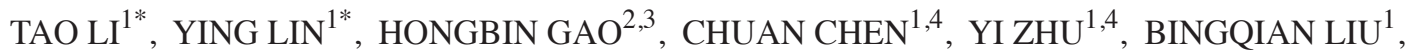 \\ YU LIAN $^{1}$, YONGHAO LI ${ }^{1}$, WENLI ZHOU ${ }^{1}$, HONGYE JIANG ${ }^{5}$, HAICHUN LI $^{1}$, QINGXIU WU ${ }^{1}$, \\ XIAOLING LIANG ${ }^{1}$, CHENJIN JIN ${ }^{1}$, XINHUA HUANG ${ }^{1}$ and LIN LU ${ }^{1}$ \\ ${ }^{1}$ State Key Laboratory of Ophthalmology, Zhongshan Ophthalmic Center, Sun Yat-sen University, \\ Guangzhou, Guangdong 510060; ${ }^{2}$ Department of Toxicology, School of Public Health and Tropical Medicine, \\ Southern Medical University, Guangzhou, Guangdong 510515; ${ }^{3}$ Guangdong Laboratory Animals Monitoring Institute, \\ Key Laboratory of Guangdong Laboratory Animals, Guangzhou, Guangdong 510663, P.R. China; \\ ${ }^{4}$ Department of Molecular and Cellular Pharmacology, University of Miami Miller School of \\ Medicine, Miami, FL 33136, USA; ${ }^{5}$ Department of Obstetrics and Gynecology, \\ The First Affiliated Hospital, Sun Yat-sen University, Guangzhou, Guangdong 510000, P.R. China
}

Received January 16, 2017; Accepted May 31, 2017

DOI: $10.3892 / \mathrm{mmr} .2017 .6887$

\begin{abstract}
Congenital macular coloboma is characterized by defined punched out atrophic lesions of the macula. The present study aimed to investigate the genetic alterations of one Chinese sporadic patient with bilateral large macular coloboma. Complete ophthalmic examinations, including best-corrected visual acuity, slit-lamp examination, fundus examination, fundus photograph and fundus fluorescein angiography imaging, Pentacam, and optical coherence tomography were performed on the patient. Genomic DNA was extracted from leukocytes in a peripheral blood sample collected from the patient, the patient's unaffected family members and from 200 unrelated control subjects from the same population. Next-generation sequencing of the known genes involved in ocular disease was performed. The functional effects of the mutation were analyzed using Polymorphism Phenotyping (PolyPhen) and Sorting Intolerant From Tolerant (SIFT). One heterozygous bestrophin 1 (BEST1) mutation c.1037C>A (p.Pro346His, p.P346H) in exon 9 and one heterozygous regulating synaptic
\end{abstract}

Correspondence to: Professor Xinhua Huang or Dr Lin Lu, State Key Laboratory of Ophthalmology, Zhongshan Ophthalmic Center, Sun Yat-sen University, 54 Xianlie South Road, Guangzhou, Guangdong 510060, P.R. China

E-mail: 1476557150@qq.com

E-mail: drlulin@126.com

*Contributed equally

Key words: macular coloboma, bestrophin 1, regulating synaptic membrane exocytosis 1 , mutation, macular atrophy membrane exocytosis 1 (RIMS1) mutation c.3481A $>\mathrm{G}$ (p.Arg1161Gly, p.R1161G) in exon 23 were identified in the patient being investigated, but not in the unaffected family members or unrelated control subjects. Polyphen and SIFT predicted that the amino acid substitution p.P346H in the BEST1 protein is damaging. In addition, Polyphen predicted that the amino acid substitution p.R1161G in the RIM1 protein is damaging. The results of the current study have increased the mutation spectrums of BEST1 and RIMS1, and are valuable for improving the current genetic counseling process and developing novel therapeutic interventions for patients with macular coloboma.

\section{Introduction}

Congenital coloboma is a very rare birth defect with a prevalence of 0.5-0.7/10,000 live births (1). Congenital macular coloboma is characterized by well-circumscribed, punched out atrophic lesions in the macula (2-4). Macular coloboma should be differentiate from other diseases, such as Best vitelliform macular dystrophy (BVMD), advanced cone-rod dystrophy (CORD), congenital toxoplasmosis macular scar, Leber's congenital amaurosis, and central areolar choroidal dystrophy $(5,6)$. It is usually sporadic, although autosomal dominant or other inheritance patterns may be followed. It is thought to be caused by the failure of normal closure of the optic fissure between 5 and 7 weeks of development (1). Macular coloboma can be classified into three types, namely pigmented macular coloboma, non-pigmented macular coloboma, and macular coloboma associated with abnormal vessels (7).

The genetic changes responsible for the pathogenesis of congenital macular coloboma are not well studied. Identification of genetic mutations in congenital macular coloboma is the first step to unravel the pathogenesis of this disease and will be helpful for genetic counseling. In this 
study, we aimed to characterize the clinical presentation of a 28 -year-old female presented with bilateral large macular coloboma, and to identify the underlying genetic changes in this patient.

\section{Patients and methods}

Study participants. One patient presented with bilateral large atrophy at the macula in both eyes underwent complete ophthalmic examinations in Zhongshan Ophthalmic Center. Visual acuity was examined using the ETDRS chart (Precision Vision, La Salle, IL, USA). Anterior segment photograph was obtained using a BX 900 Slit Lamp (Haag-Streit, Bern, Switzerland). Anterior segment measurements were taken by Pentacam HR version 70700 (Oculus, Wetzlar, Germany). Fundus photograph were carried out using a Heidelberg Retina Angiograph (Heidelberg Engineering, Inc., Heidelberg, Germany). OCT was carried out by Cirrus HD-OCT (Carl Zeiss Meditec, Inc., Dublin, CA, USA). Physical examinations were performed to exclude systemic diseases. Venous blood samples from this patient, her unaffected family members, and 200 unrelated control subjects from the same population were collected.

Target capture and next-generation sequencing. A capture panel of inherited retinal-disease genes was previously designed and assessed by our group. The capture panel comprised 708,919 bp that covered all exons together with the flanking exon and intron boundaries $( \pm 15$ bp) of 175 genes, including 138 genes causing common inherited nonsyndromic eye diseases and 54 genes causing syndromic eye diseases that have been previously reported and have been accepted by researcheres in this field.

Genomic DNA from peripheral blood leucocytes was extracted using the QIAamp DNABlood Midi Kit (Qiagen, Hilden, Germany). Then the genomic DNA was fragmented by Covaris LE220 (Covaris, Inc., Woburn, MA, USA) to generate paired-end library (200-250 bp). The library was enriched by array hybridization as previously described (8), followed by elution and post-capture amplification. The products were then subjected to Agilent 2100 Bioanalyzer and ABI StepOne for estimating the magnitude of enrichment. After quality control, captured library sequencing was carried out on Illumina HiSeq2500 Analyzers (Illumina, San Diego, CA, USA) for 90 cycles per read to generate paired-end reads. Image analysis, error estimation, and base calling were performed using Illumina Pipeline software (version 1.3.4) to generate raw data.

Data analysis and interpretation of genetic variants. To detect the potential variants in the family, we performed bioinformatics processing and data analysis after receiving the primary sequencing data. We used previously published filtering criteria to generate 'clean reads' for further analysis (8). The 'clean reads' (with a length of $90 \mathrm{bp}$ ) derived from targeted sequencing and filtering were then aligned to the human genome reference (hg19) using the BWA (Burrows Wheeler Aligner) Multi-Vision software package (9). After alignment, the output files were used to perform sequencing coverage and depth analysis of the target region, single-nucleotidevariants (SNVs) and INDEL calling. We used SOAPsnp software (9) and Samtools (10) to detect SNVs and indels. All SNVs and indels were filtered and estimated via multiple databases, including NCBI dbSNP, HapMap, 1,000 human genome dataset and a database of 200 Chinese healthy adults.

To predict the effect of missense variants, SIFT and PolyPhen were used to predict the possible impact of an amino acid substitution on the protein structure and function using straightforward physical and comparative considerations. Variants were predicted to be pathogenic only when at least one of the two programs predicted deleterious effect of the amino acid substitution on the protein structure and function. The Human Gene Mutation Database (HGMD) was used to screen mutations reported in published studies.

We also used PolyPhen to check whether the mutations affected highly conserved amino acid residues.

Mutation validation. The two novel pathogenic mutations were validated using conventional polymerase chain reaction (PCR) -based sequencing methods (11-13). Exon 9 of the BEST1 gene and the Exon 23 of RIMS1 were amplified by PCR with respective primers (Table I). Briefly, PCR was conducted in $50 \mu \mathrm{l}$ reactions. The cycling profile included one cycle at $94^{\circ} \mathrm{C}$ for $5 \mathrm{~min}$, followed by 40 cycles at $94^{\circ} \mathrm{C}$ for $45 \mathrm{sec}, 59-60^{\circ} \mathrm{C}$ for $45 \mathrm{sec}, 72^{\circ} \mathrm{C}$ for $45 \mathrm{sec}$, and one cycle at $72^{\circ} \mathrm{C}$ for $10 \mathrm{~min}$. The PCR products were sequenced from both directions with an ABI3730 Automated Sequencer (PE Biosystems, Foster City, CA, USA). The sequencing results were analyzed using Seqman (version 2.3; Technelysium Pty, Ltd., Brisbane, QLD, Australia), and compared with the reference sequences in the database at the National Center for Biotechnology Information.

All experimental protocols were carried out according to the guidelines approved by the Ethics Committee of Zhongshan Ophthalmic Center, and in accordance with the Declaration of Helsinki. Informed consent was obtained from all subjects. The data generated or analyzed in the current study are included herein.

\section{Results}

Clinical data. The patient studied in this report was from the southern area of China. Patient is a 28 -year-old female without known familial history of ocular disease. Her best-corrected visual acuity was $1.3 \operatorname{LogMAR}$ in the right eye, and figure count $/ 40 \mathrm{~cm}(\mathrm{FC} / 40 \mathrm{~cm})$ in the left eye. Anterior segment photograph showed some opacities in the lens of both eyes (Fig. 1). Fundus examination revealed bilateral large atrophy in the macula of each eye with well-circumscribed borders (Fig. 2). OCT showed that the foveal region of both eyes were abnormally thin. A large cave in the macular area and retinal schisis were observed in the left eye (Fig. 3).

Mutation screening. A heterozygous BEST1 mutation c.1037C >A (p.Pro346His, p.P346H) in exon 9 and a heterozygous RIMS1 mutation c.3481A $>\mathrm{G}$ (p.Arg1161Gly, p.R1161G) in exon 23 were identified in the affected case, but not in any of the normal controls (Fig. 4). The first mutation we identified has previously been reported in Japanese 
Table I. Primers used for the amplification of the BEST1 and RIMS1 in this study.

\begin{tabular}{lrcccc}
\hline Gene & Exon & \multicolumn{1}{c}{ Forward (5'-3') } & Reverse $\left(5^{\prime}-3^{\prime}\right)$ & $\begin{array}{c}\text { Product } \\
\text { Annealing } \\
\text { size } \\
(\mathrm{bp})\end{array}$ & $\begin{array}{c}\text { temperature } \\
\left({ }^{\circ} \mathrm{C}\right)\end{array}$ \\
\hline BEST1 & 9 & CAGGGAAACTGAGGTCCAGA & AGGCTGTCCTTCGAGTAGCA & 539 & 60 \\
RIMS1 & 23 & GGCGGATTCCAAACATCTTCC & AGGTGCTTTACCAGAGTTGGC & 487 \\
\hline
\end{tabular}
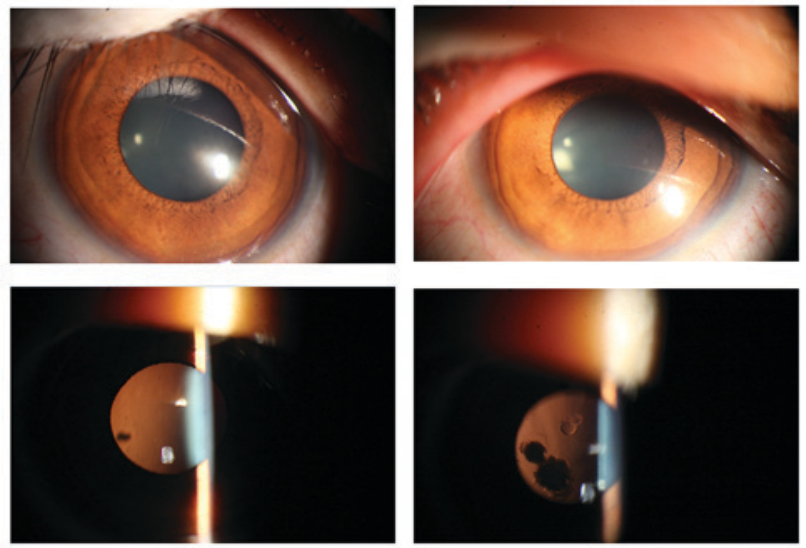

$O D$

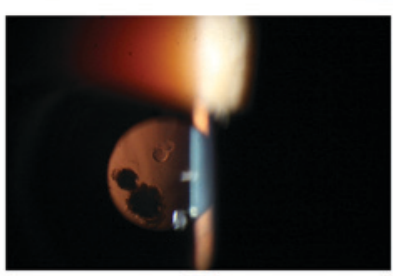

os

Figure 1. Anterior segment photograph showed some opacities in the lens of both eyes.
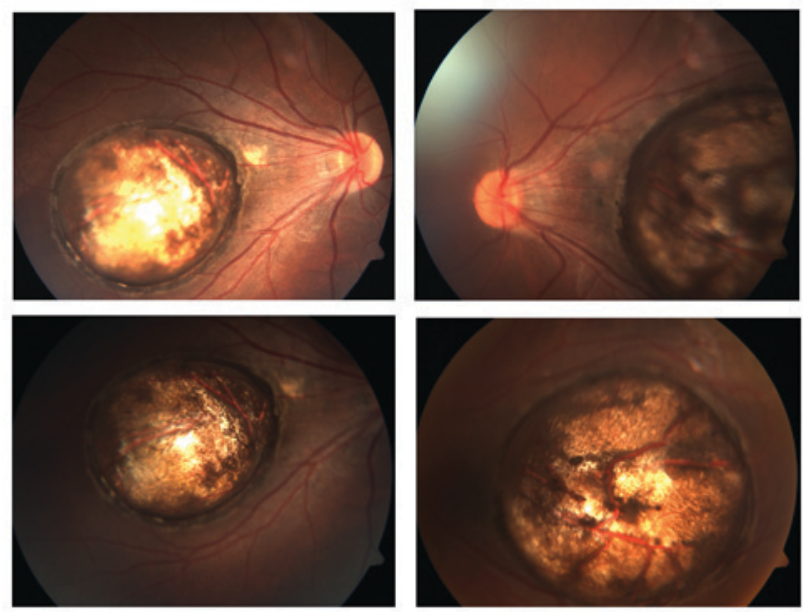

OD

OS

Figure 2. Fundus examination revealed bilateral large atrophy in the macula of each eye with well-circumscribed borders.

patients (14). Since we were unable to obtain information of the patient's parents, we could not determine the inheritance pattern of the mutations. Polyphen and SIFT predicted that the amino acid substitution p.P346H in protein bestrophin 1 is damaging (Fig. 5), and Polyphen predicted that the amino acid substitution p.R1161G in protein RIM1 is damaging (Fig. 6). Multiple sequenced alignment indicated that the residue at position 346 of bestrophin-1 and the residue at position 1161 of RIM1 are highly conserved across species.

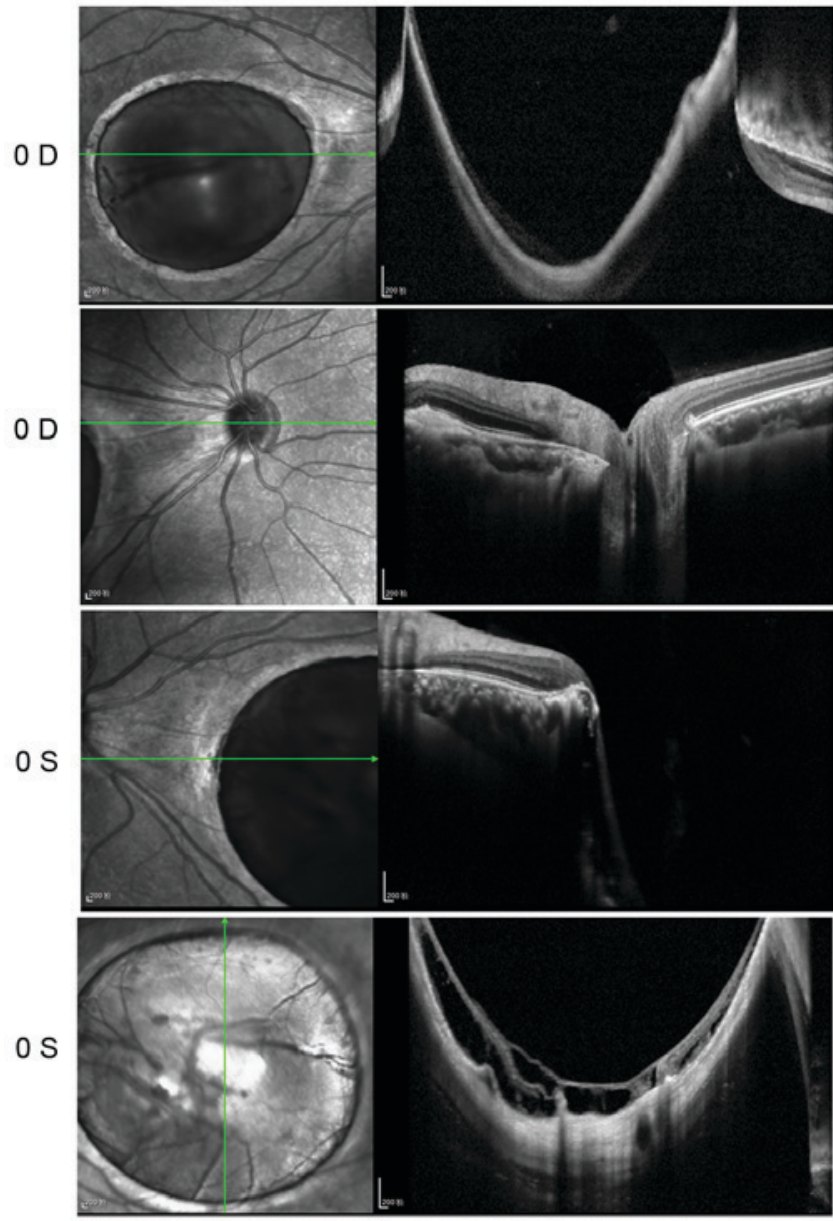

Figure 3. OCT showed that the foveal region of both eyes were abnormally thin. A large cave in the macular area and retinal schisis were observed in the left eye.

\section{Discussion}

Macular coloboma may result from intrauterine inflammation (15), and can be associated with systemic developmental abnormalities. Notably, it may be difficult to distinguish macular coloboma with macular atrophy if medical history is not provided. The underlying biological mechanism for development of macular coloboma is unclear. Interestingly, we found that this patient had two different mutations simultaneously, reminding us that some serious congenital defects can be caused by multiple mutations on multiple genes, making gene therapy more challenging.

BVMD is one of the most frequent form of autosomal dominant macular dystrophy (16). It is associated 

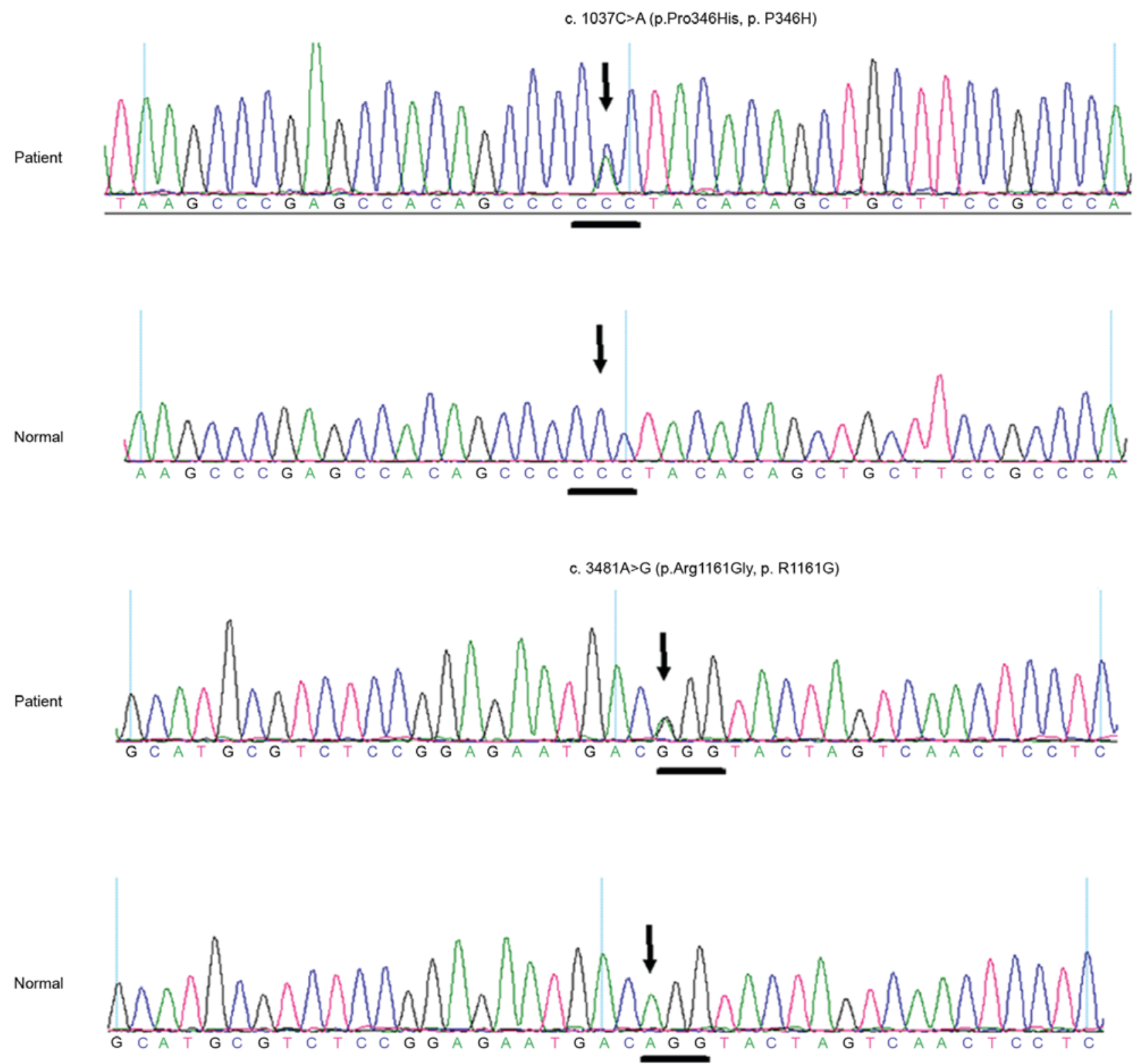

Figure 4. A heterozygous BEST1 mutation c.1037C >A (p.Pro346His) in exon 9 and a heterozygous RIMS1 mutation c.3481A>G (p.Arg1161Gly) in exon 23 were identified in the affected case, but not in the unaffected family members or unrelated control subjects.

with mutations in the BEST1 gene (17) and results from dysfunction of the retinal pigment epithelium (RPE) (18). Bestrophin-1 is the product of the gene BEST1. This protein is mainly expressed in the basolateral plasma membrane of the RPE (19). This protein contains several domains with a high degree of evolutionary conservation. The function of Bestrophin-1 remains unclear, and some studies proposed that it acts as a $\mathrm{Cl}^{-}$channel activated by intracellular $\mathrm{Ca}^{2+}$ and/or as a channel regulator $(20,21)$. A previous study conducted by Katagiri et al (14). identified a novel mutation p.P346H on BEST1 in a 38-year-old patient who was in the vitelliruptive stage, which was less serious than the patient in our study. It is likely that in BVMD, the clinical manifestations of transheterozygous mutations may be more serious than a single mutation.

CORD is one of the common forms of inherited retinal degeneration with a prevalence of $1 / 40000(22,23)$. CORD is characterized by the impairment of cone photoreceptors with or without dysfunction of rod photoreceptors $(24,25)$. Clinical manifestations in CORD include photophobia, reduced visual acuity, color vision defects, and central scotomata (26). At present, a total of 30 genes have been found associated with CORD, including 10 genes related to autosomal dominant CORD (AIPL1, CRX, GUCAIA, GUCY2D, PITPNM3, PROM1, PRPH2, RIMS1, SEMA4A, UNC119) (27,28). RIM1, the protein product of RIMS1, localizes to the presynaptic active zones in brain and retinal tissue, and plays an important role in regulating synaptic vesicle release and presynaptic plasticity (29). RIM1 is a large multi-domain protein that interacts with multiple molecules at different regions (30).

In this case, we consider the patient had BVMD and CORD simultaneously. Mutations on BEST1 and RIM1 affected the RPE and photoreceptors, respectively. Therefore, the atrophy of the retina was very serious with only a little retina tissue remained. For these patients, transplantation of the retina 


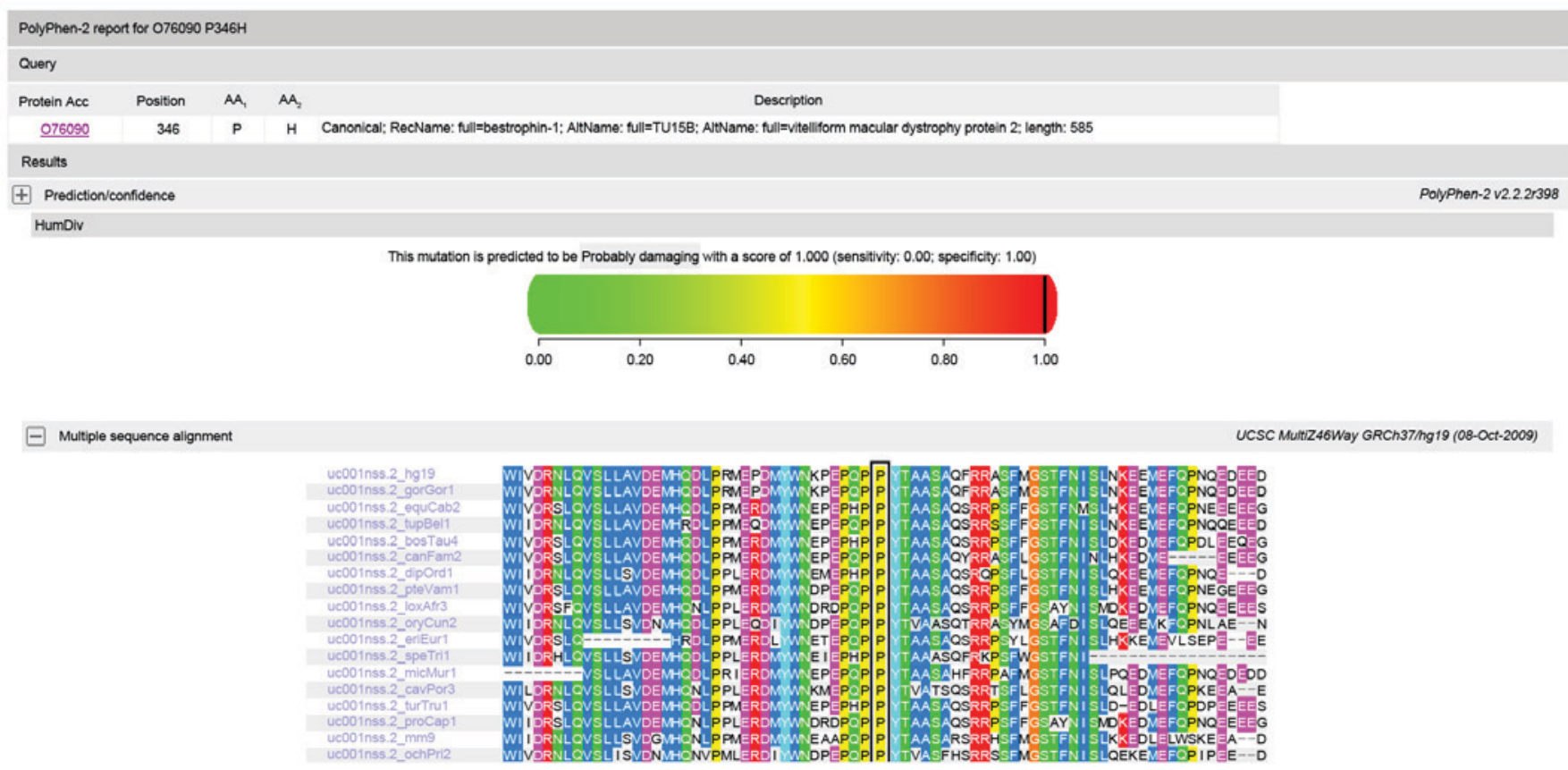

Figure 5. Polyphen and SIFT predicted that the amino acid substitution p.P346H in protein bestrophin 1 is damaging. Multiple sequenced alignment (Basic Local Alignment Search Tool) indicated that the residue at position 346 of bestrophin-1 is highly conserved across species.

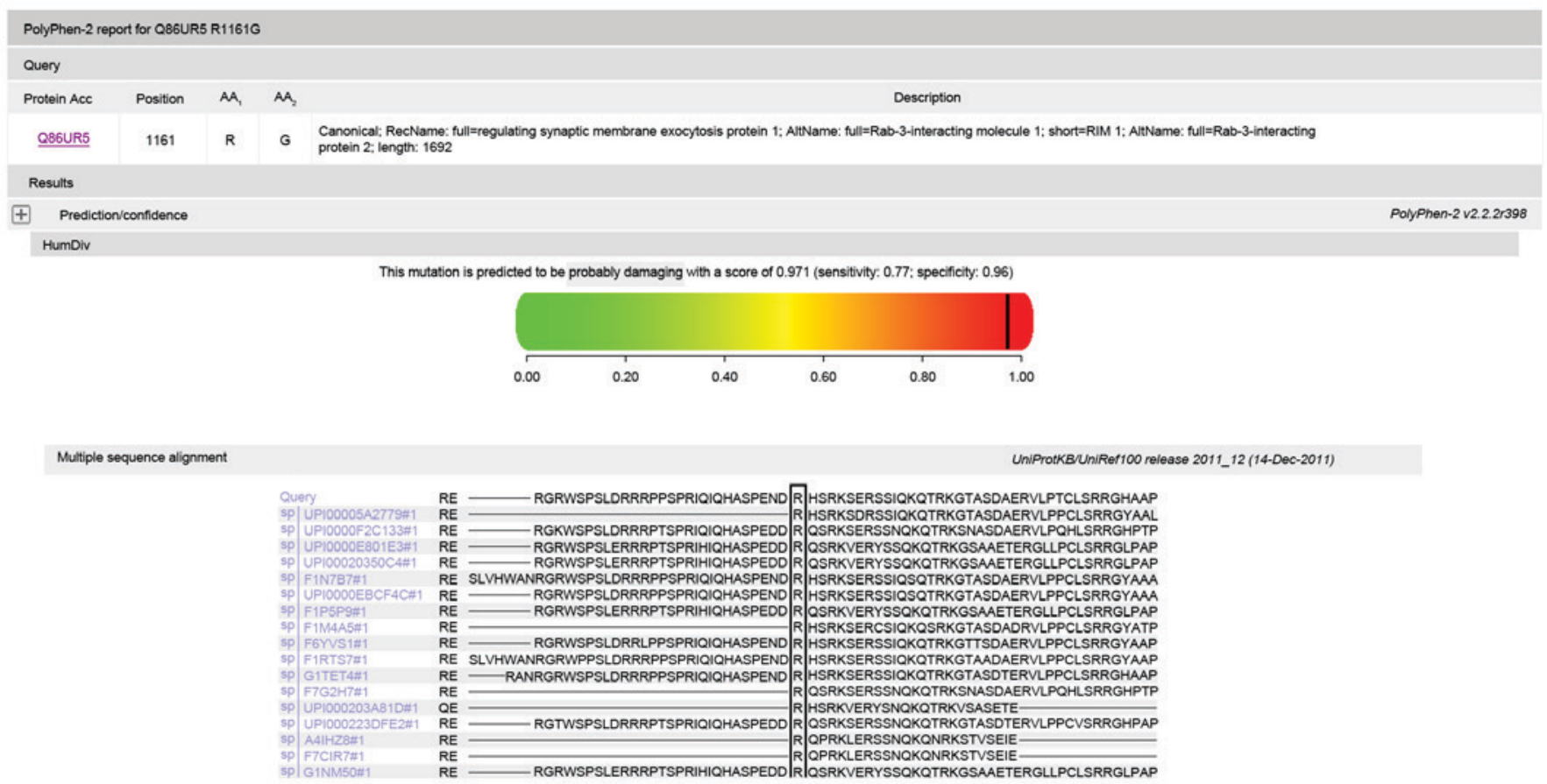

Figure 6. Polyphen predicted that the amino acid substitution p.R1161G in protein RIM1 is damaging. The residue at position 1161 of RIM1 is highly conserved across species.

stem cells or retina cell membrane may be more feasible for treatment.

In summary, our study identified two mutations of BESTI and RIMS1 in one Chinese patient with bilateral macular coloboma. These findings expand the mutation spectrums of BESTI and RIMS1, and will be valuable for genetic counseling and development of therapeutic interventions for patients with macular coloboma.

\section{Acknowledgements}

The authors are grateful to all of the patients, their families, and the control volunteers for participating in this study. This study was supported by the National Natural Science Foundation of China (grant nos. 81500709, 81570862, 81371019 and 81670872), the Medical Scientific Research Foundation of Guangdong Province (grant no. A2016460), 
and the Fundamental Research Funds for the Universities (grant no. 13ykpy43).

\section{References}

1. Hornby SJ, Adolph S, Gilbert CE, Dandona L and Foster A Visual acuity in children with coloboma: Clinical features and a new phenotypic classification system. Ophthalmology 107: $511-520,2000$

2. Varghese M, Kavalakatt JA, Pandey S and Kolath JJ: Macular coloboma. Oman J Ophthalmol 9: 67-68, 2016.

3. Primo SA: Macular coloboma. J Am Optom Assoc 61: 373-377, 1990.

4. Sharma S, Naqvi A and Cruess AF: Bilateral macular colobomas. Can J Ophthalmol 31: 27-28, 1996.

5. Ishaq M, Mukhtar A and Khan S: Macular coloboma in a child with usher syndrome. J Ayub Med Coll Abbottabad 27: 470-472, 2015

6. Izumikawa Y: Macular coloboma-brachydactyly. Ryoikibetsu Shokogun Shirizu (34 Pt 2): 126-127, 2001 (In Japanese).

7. Jimenez-Sierra JM, Ogden TE and Van Boemel GB: Inherited retinal diseases. A diagnostic guide. The C. V. Mosby Company, St. Louis, 1989.

8. Wei X, Ju X, Yi X, Zhu Q, Qu N, Liu T, Chen Y, Jiang H, Yang $\mathrm{G}$, Zhen $\mathrm{R}$, et al: Identification of sequence variants in genetic disease-causing genes using targeted next-generation sequencing. PLoS One 6: e29500, 2011.

9. Li R, Li Y, Fang X, Yang H, Wang J, Kristiansen $\mathrm{K}$ and Wang J: SNP detection for massively parallel whole-genome resequencing. Genome Res 19: 1124-1132, 2009.

10. Li H, Handsaker B, Wysoker A, Fennell T, Ruan J, Homer N, Marth G, Abecasis G and Durbin R; 1000 Genome Project Data Processing Subgroup: The sequence alignment/map format and SAMtools. Bioinformatics 25: 2078-2079, 2009.

11. Lin Y, Liu X, Yu S, Luo L, Liang X, Wang Z, Chen C, Zhu Y, Ye S, Yan $\mathrm{H}$ and Liu Y: PAX6 analysis of two sporadic patients from southern China with classic aniridia. Mol Vis 18 : 2190-2194, 2012.

12. Lin Y, Liang X, Ai S, Chen C, Liu X, Luo L, Ye S, Li B, Liu Y and Yang H: FGFR2 molecular analysis and related clinical findings in one Chinese family with Crouzon syndrome. Mol Vis 18: 449-454, 2012.

13. Lin Y, Ai S, Chen C, Liu X, Luo L, Ye S, Liang X, Zhu Y, Yang $\mathrm{H}$ and Liu Y: Ala344Pro mutation in the FGFR2 gene and related clinical findings in one Chinese family with Crouzon syndrome. Mol Vis 18: 1278-1282, 2012.

14. Katagiri S, Hayashi T, Ohkuma Y, Sekiryu T, Takeuchi T, Gekka T, Kondo M, Iwata T and Tsuneoka H: Mutation analysis of BEST1 in Japanese patients with Best's vitelliform macular dystrophy. Br J Ophthalmol 99: 1577-1582, 2015.

15. Yamaguchi $\mathrm{K}$ and Tamai M: Congenital macular coloboma in Down syndrome. Ann Ophthalmol 22: 222-223, 1990.
16. Low S, Davidson AE, Holder GE, Hogg CR, Bhattacharya SS, Black GC, Foster PJ and Webster AR: Autosomal dominant best disease with an unusual electrooculographic light rise and risk of angle-closure glaucoma: A clinical and molecular genetic study. Mol Vis 17: 2272-2282, 2011.

17. Tian R, Yang G, Wang J and Chen Y: Screening for BEST1 gene mutations in Chinese patients with bestrophinopathy. Mol Vis 20: 1594-1604, 2014.

18. Wong RL, Hou P, Choy KW, Chiang SW, Tam PO, Li H, Chan WM, Lam DS, Pang CP and Lai TY: Novel and homozygous BEST1 mutations in Chinese patients with Best vitelliform macular dystrophy. Retina 30: 820-827, 2010.

19. Lin Y, Gao H, Liu Y, Liang X, Liu X, Wang Z, Zhang W, Chen J, Lin Z, Huang $X$ and Liu Y: Two novel mutations in the bestrophin-1 gene and associated clinical observations in patients with best vitelliform macular dystrophy. Mol Med Rep 12: 2584-2588, 2015.

20. Lin CF and Sarraf D: Best disease presenting as a giant serous pigment epithelial detachment. Retin Cases Brief Rep 8: 247-250, 2014

21. Apushkin MA, Fishman GA, Taylor CM and Stone EM: Novel de novo mutation in a patient with best macular dystrophy. Arch Ophthalmol 124: 887-889, 2006.

22. Hamel CP: Cone rod dystrophies. Orphanet J Rare Dis 2: 7, 2007.

23. Brodie S: Cone-rod dystrophy in Danon disease. Graefes Arch Clin Exp Ophthalmol 250: 633, 2012.

24. Burstedt MS, Ristoff E, Larsson A and Wachtmeister L: Rod-cone dystrophy with maculopathy in genetic glutathione synthetase deficiency: A morphologic and electrophysiologic study. Ophthalmology 116: 324-331, 2009.

25. Khan AO and Abu-Safieh L: Rod-Cone dystrophy with initially preserved visual acuity despite early macular involvement suggests recessive CERKL mutations. Ophthalmic Genet 36: 369-372, 2015

26. Kuehlewein L and Sadda SR: Rod-Cone dystrophy associated with williams syndrome. Retin Cases Brief Rep 9: 298-301, 2015.

27. Pras E, Abu A, Rotenstreich Y, Avni I, Reish O, Morad Y, Reznik-Wolf $\mathrm{H}$ and Pras E: Cone-rod dystrophy and a frameshift mutation in the PROM1 gene. Mol Vis 15: 1709-1716, 2009.

28. Huang L, Xiao X, Li S, Jia X, Wang P, Sun W, Xu Y, Xin W, Guo X and Zhang Q: Molecular genetics of cone-rod dystrophy in Chinese patients: New data from 61 probands and mutation overview of 163 probands. Exp Eye Res 146: 252-258, 2016.

29. Weiss N, Sandoval A, Kyonaka S, Felix R, Mori Y and De Waard M: Rim1 modulates direct G-protein regulation of $\mathrm{Ca}(\mathrm{v}) 2.2$ channels. Pflugers Arch 461: 447-459, 2011.

30. Schoch S, Mittelstaedt T, Kaeser PS, Padgett D, Feldmann N, Chevaleyre V, Castillo PE, Hammer RE, Han W, Schmitz F, et al: Redundant functions of RIM1alpha and RIM2alpha in $\mathrm{Ca}(2+)$-triggered neurotransmitter release. EMBO J 25: 5852-5863, 2006. 